\title{
A model assisted ionospheric electron density reconstruction method based on vertical TEC data ingestion
}

\author{
Bruno Nava $\left({ }^{1}\right)$, Pierdavide Coïsson $\left({ }^{1}\right)$, Gloria Miró Amarante $\left(^{1}\right)$, \\ Francisco Azpilicueta $\left({ }^{2}\right)$ and Sandro M. Radicella $\left({ }^{1}\right)$ \\ ( $\left.{ }^{1}\right)$ The Abdus Salam International Centre for Theoretical Physics (ICTP), Trieste, Italy \\ $\left(^{2}\right)$ Facultad de Ciencias Astronómicas y Geofísicas, Universidad Nacional de La Plata, Argentina
}

\begin{abstract}
A technique to reconstruct the electron density of the ionosphere starting from total electron content values has been developed using the NeQuick ionospheric electron density model driven by its effective ionization parameter $A z$. The technique is based on the computation of $A z$ values for a suitable worldwide grid of points. A simple way to obtain relevant $A z$ grids is to use global vertical Total Electron Content (TEC) maps to define for each grid point as $A z$ value, the one that minimizes the difference between the experimental and the modeled vertical TEC. Having a global grid of $A z$ values it is possible to compute the electron density at any point in the ionosphere using NeQuick. As a consequence, slant TEC values for specific ground station to satellite links or ionosphere peak parameter values at any location can be calculated. The results of the comparisons between experimental and reconstructed slant TEC as well as experimental and reconstructed peak parameters values indicate that the proposed reconstruction method can be used to reproduce the observed ionosphere in a realistic way.
\end{abstract}

Key words ionospheric data ingestion - electron density model - total electron content

\section{Introduction}

Like other empirical ionospheric models (e.g., International Reference Ionosphere - IRI: Bilitza, 2001) NeQuick has been conceived to reproduce median values of the ionosphere electron density. In order to improve its capabilities to describe the ionosphere for actual conditions, a technique based on experimental Total Electron Content (TEC) data ingestion into the model has been developed. The technique

Mailing address: Dr. Bruno Nava, The Abdus Salam International Centre for Theoretical Physics (ICTP), Strada Costiera 11, 34014 Trieste, Italy; e-mail: bnava@ictp.it considers global vertical TEC maps to determine the corresponding global grids of the NeQuick driving parameter $A z$. When the $A z$ grid is computed, NeQuick model is able to calculate the 3D electron density of the ionosphere and therefore the total electron content value for any given ray-path can be calculated by means of numerical integration. The emphasis has been put on TEC because it is directly related to the range delay in trans-ionospheric propagation and therefore such kind of reconstruction method could have an application related to satellite navigation systems.

It must be noted that other techniques to reconstruct the electron density of the ionosphere have been developed. They are of different complexity and can rely on several kinds of models. The Global Assimilative Ionospheric Model (Wang et al., 2004), for example, is based on assimilation of data originating from different sources and implies the use of first 
principle models. On the contrary, the proposed reconstruction method aims to be simpler, being based on the use of an empirical electron density model and it foresees the ingestion of only TEC data that, for instance, are the most widely available ionospheric data.

\section{NeQuick model}

The NeQuick model is an ionospheric electron density model developed at the Aeronomy and Radiopropagation Laboratory of The Abdus Salam International Centre for Theoretical Physics (ICTP), Trieste, Italy and at the Institute for Geophysics, Astrophysics and Meteorology of the University of Graz, Austria, in the framework of the European Commission COST (Co-operation in the field of Scientific and Technical Research) Action 251. It is based on the original «profiler» proposed by Di Giovanni and Radicella (DGR, Di Giovanni and Radicella, 1990). NeQuick is able to give the electron concentration distribution on both the bottomside and topside of the ionosphere and it is a quick-run model particularly tailored for trans-ionospheric applications. The model has been used by the European Space Agency EGNOS (European Geostationary Navigation Overlay Service) Project for assessment analysis and has been proposed for single-frequency positioning operations in the framework of the European Galileo Project. It has been adopted by the International Telecommunication Union, Radiocommunication sector (ITU-R) Recommendation P.531-6 (now superseded by P.5317; ITU, 2001) as a suitable method for TEC modeling. To describe the electron density of the ionosphere above $100 \mathrm{~km}$ and up to the $F_{2}$ layer peak this model uses a modified DGR profile formulation (Radicella and Leitinger, 2001) which includes five semi-Epstein layers with modeled thickness parameters and is based on anchor points defined by $f_{o} E, f_{o} F_{1}$, $f_{o} F_{2}$ and $M(3000) F_{2}$ values (Hochegger et al., 2000). These values can be modeled or experimentally derived. The model topside is represented by a semi-Epstein layer with a height dependent thickness parameter empirically determined.
The basic inputs of NeQuick electron density model are: position, time and solar flux (or sunspot number); the output is the electron concentration at the given location and time. In addition the NeQuick package includes specific routines to evaluate the electron density along any ray-path and the corresponding TEC by numerical integration. NeQuick (FORTRAN 77) source code is available at http://www.itu.int/ITU-R/software/studygroups/rsg3/databanks/ionosph/

\subsection{Reconstruction technique}

For the purpose of the present work, NeQuick has to be regarded as a «profiler» driven by its local ionization level index that plays the role of an effective $10.7 \mathrm{~cm}$ solar radio flux and determines the complete vertical electron density profile at a given epoch and for a given location. Being the vertical TEC calculated with NeQuick a monotonic function of this index alone, it is possible to define as $A z$ the ionization level value that minimizes the difference between an experimental vertical TEC and the corresponding modeled vertical TEC obtained by integration of the vertical electron density profile.

Applying this concept to all vertical TEC values of a global experimental vertical TEC map, it is possible to retrieve an $A z$ grid that drives NeQuick to generate a $3 \mathrm{D}$ representation of the ionosphere over the globe and reproduces the starting vertical TEC map with the requested accuracy. The same approach was previously used in Nava et al. (2003), while similar concepts have been adopted in Komjathy et al. (1998).

In order to apply the described procedure, NeQuick code has been adapted to use global $A z$ grids as input. The grid points are spaced $2.5^{\circ}$ in latitude and $5^{\circ}$ in longitude and are interpolated to retrieve $A z$ values at any wanted geographic location. Under suitable conditions, $A z$ grids for different epochs can also be interpolated in time to obtain new grids for epochs when experimental data are not available.

An operational application of this reconstruction method was implemented using available experimental vertical TEC maps (Nava et al., 2003). Several institutions around the world pro- 
duce such maps using Global Positioning System (GPS) derived slant TEC data: the network of the International GPS Service (IGS) stations in fact provides measurements that can be used to obtain information on ionospheric electron content along the ray-paths from satellites to ground receivers. For example the Centre for Orbits DEterminations (CODE), University of Berne, Switzerland, produces global bi-hourly maps (Schaer et al., 1996) based on spherical harmonics expansion of vertical TEC. These maps are given on a daily basis and made available through the Internet (http://www.cx.unibe.ch/aiub/ionosphere.html).

For the purpose of the present study the vertical TEC maps produced by the Satellite and Geodesy Group of the National University of La Plata, Argentina, have been used as reference for the minimization process. La Plata vertical TEC maps are computed using GPS data collected from an average of about 130 IGS stations distributed worldwide. La Plata maps are based on a spherical harmonic expansion of degree and order 15 for vertical TEC and the daily solution consists of 24 sets of coefficients and one instrumental delay value for each station and for each satellite included in the computation (Brunini, 1998). La Plata maps have been chosen because they are computed on hourly basis and they are built using the sun fixed/Modip reference frame, Modip $\mu$ being defined by

$$
\tan \mu=\frac{\phi}{\sqrt{\cos \phi}}
$$

with $\phi$ the true magnetic dip in the ionosphere (usually at $300 \mathrm{~km}$ ) and $\phi$ the geographic latitude (Rawer, 1963).

These features improve temporal and spatial resolution of the vertical TEC maps and then slightly better results are expected when NeQuick is used together with the reconstruction technique to reproduce the electron density of the ionosphere. An example of equivalent vertical TEC for the day 27 March 2000 at 17:00 UT is shown in fig. 1.

\subsection{Test of the technique}

In order to evaluate the effectiveness of the reconstruction technique, slant TEC and vertical incidence experimental data have been used for comparison with the corresponding reconstructed quantities.

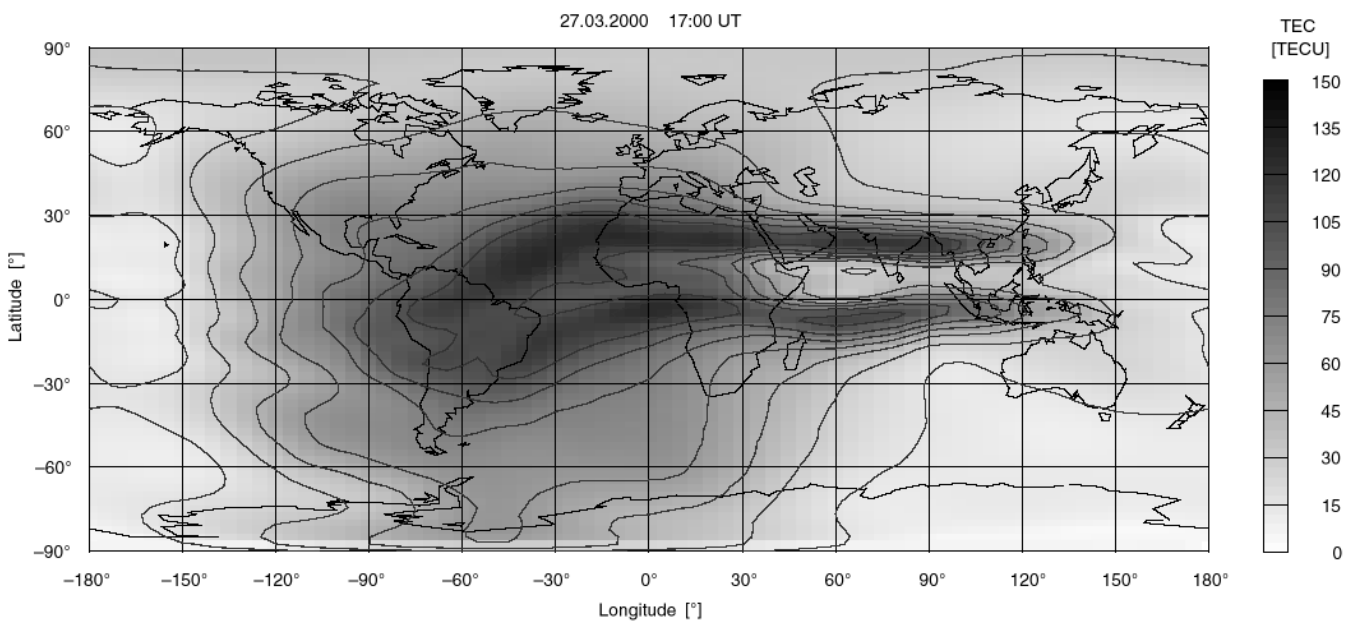

Fig. 1. Example of global vertical TEC map produced with La Plata technique for the day 27 March 2000, 17:00 UT. 
The day 27 March 2000 (a quiet day in a period of high solar activity) was chosen as reference day for the test and hourly La Plata maps were used to generate the set of $24 \mathrm{Az}$ grids needed to drive the NeQuick model.
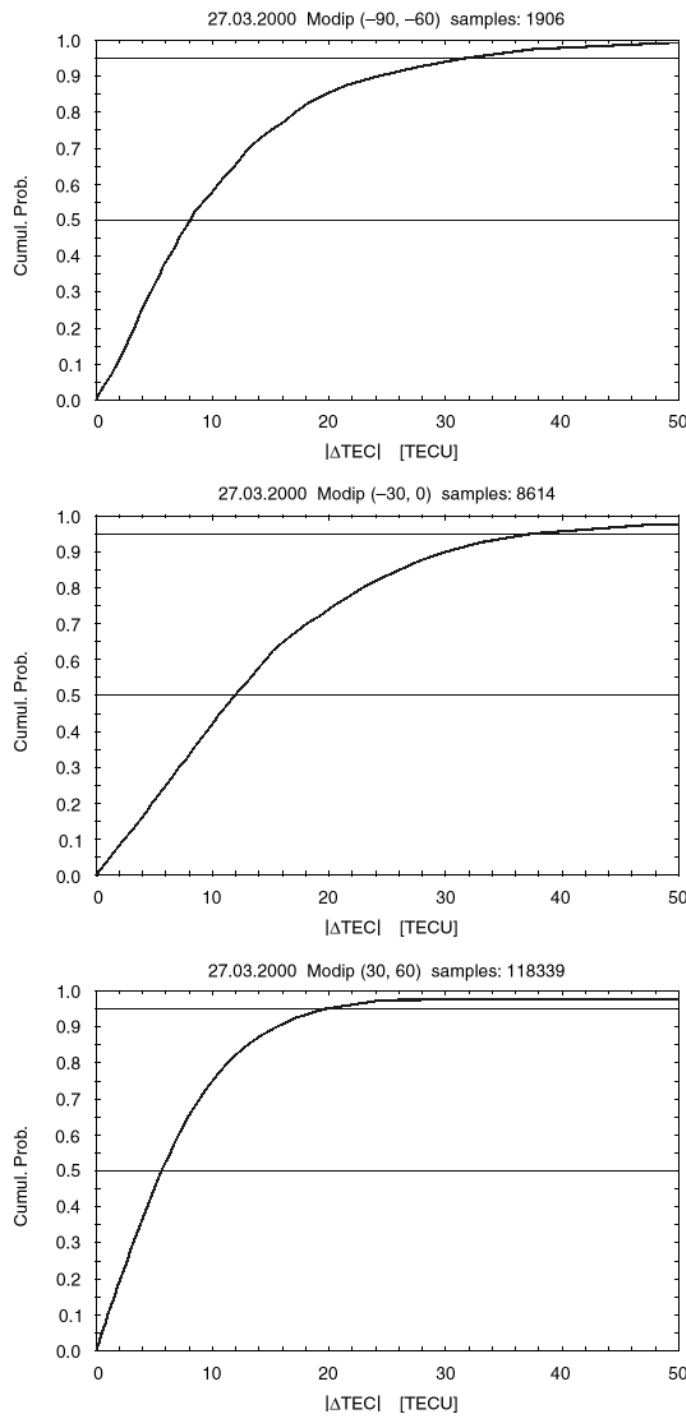

\subsection{Slant TEC}

For consistency reasons, the slant TEC data used to validate the reconstruction technique were computed from RINEX files recorded at
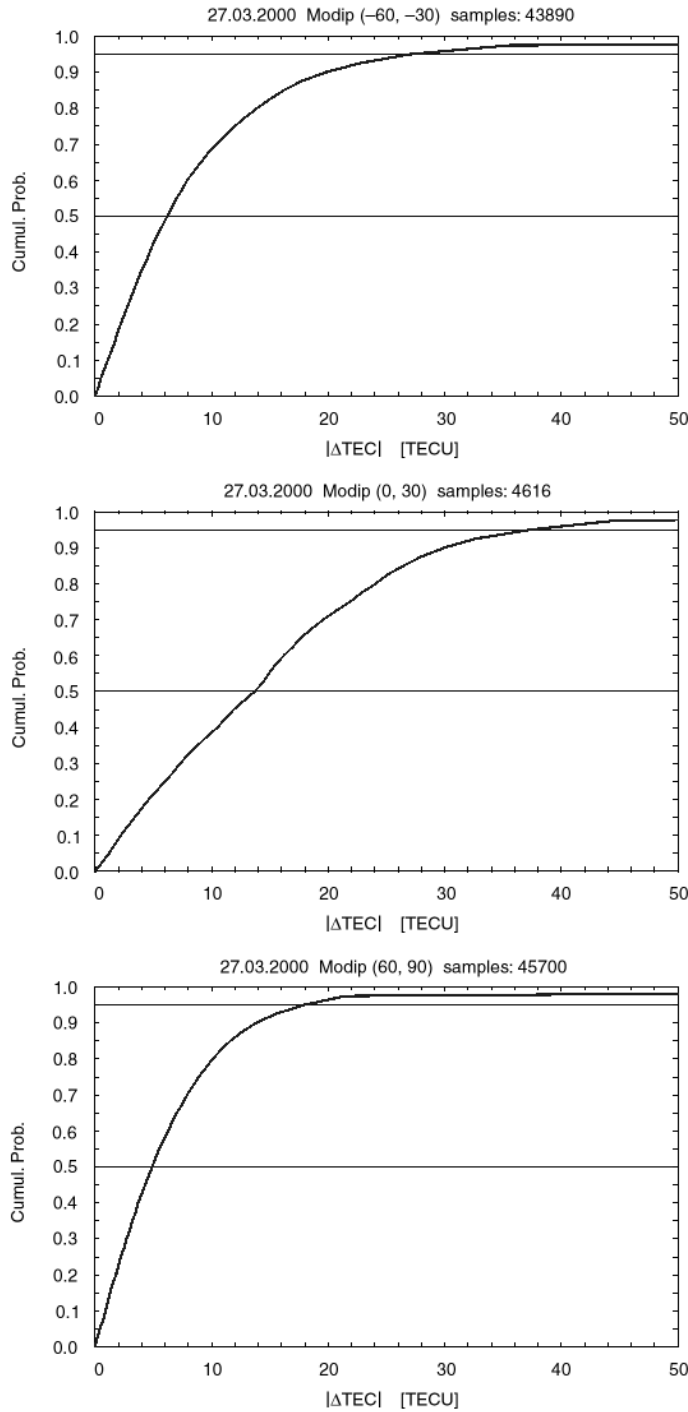

Fig. 2. Cumulative distributions of the absolute values of the differences between experimental and reconstructed slant TEC data for stations lying in different Modip intervals. The statistics are computed using slant TEC data at 5 min time intervals for the day 27 March 2000. 
the same IGS stations used to obtain the vertical TEC maps. An elevation mask of $10^{\circ}$ to all available satellites was applied and for practical purposes slant TEC data every $5 \mathrm{~min}$ were used. The experimental slant TEC data were compared with the corresponding slant TEC values com-

Table I. Statistical analysis summary for the day 27 March 2000: the first and second columns indicate the Modip interval with the corresponding number of IGS stations whose data were used to compute the cumulative distribution of the absolute value of the errors; the third column indicates the 95 percentile of the distribution of the absolute value of the errors.

\begin{tabular}{ccc}
\hline \hline Modip interval & Number of stations & 95 percentile \\
\hline$\left(-90^{\circ},-60^{\circ}\right)$ & 2 & $31.6 \mathrm{TECU}$ \\
$\left(-60^{\circ},-30^{\circ}\right)$ & 25 & $27.0 \mathrm{TECU}$ \\
$\left(-30^{\circ}, 0^{\circ}\right)$ & 5 & $37.4 \mathrm{TECU}$ \\
$\left(0^{\circ}, 30^{\circ}\right)$ & 4 & $37.4 \mathrm{TECU}$ \\
$\left(30^{\circ}, 60^{\circ}\right)$ & 72 & $19.6 \mathrm{TECU}$ \\
$\left(60^{\circ}, 90^{\circ}\right)$ & 24 & $17.9 \mathrm{TECU}$ \\
\hline
\end{tabular}

puted with NeQuick driven by $A z$ grids derived from La Plata vertical TEC maps. Therefore $A z$ values at 5 min time interval were calculated by interpolation of consecutive hourly $A z$ values.

Defining as error the difference between a reconstructed and the corresponding experimental slant TEC, for each IGS station the data analysis was based on the computation of cumulative distributions of the absolute values of the errors obtained with $24 \mathrm{~h}$ of data. In order to have a global view of the reconstruction technique capabilities, cumulative distributions of the absolute values of the errors were computed taking into account data for stations lying in specific Modip intervals. These results are shown in fig. 2, where the cumulative distributions of the absolute values of the errors are plotted together with the lines corresponding to 50 and 95 percentiles. The statistics are summarized in table I, where the number of stations considered and the value in TECU $\left(1\right.$ TECU $\left.=10^{16} \mathrm{~m}^{-2}\right)$ corresponding to the 95 percentile for each Modip interval are indicated.

The analyzed data show a general asymmetry of errors behavior in the two hemispheres of

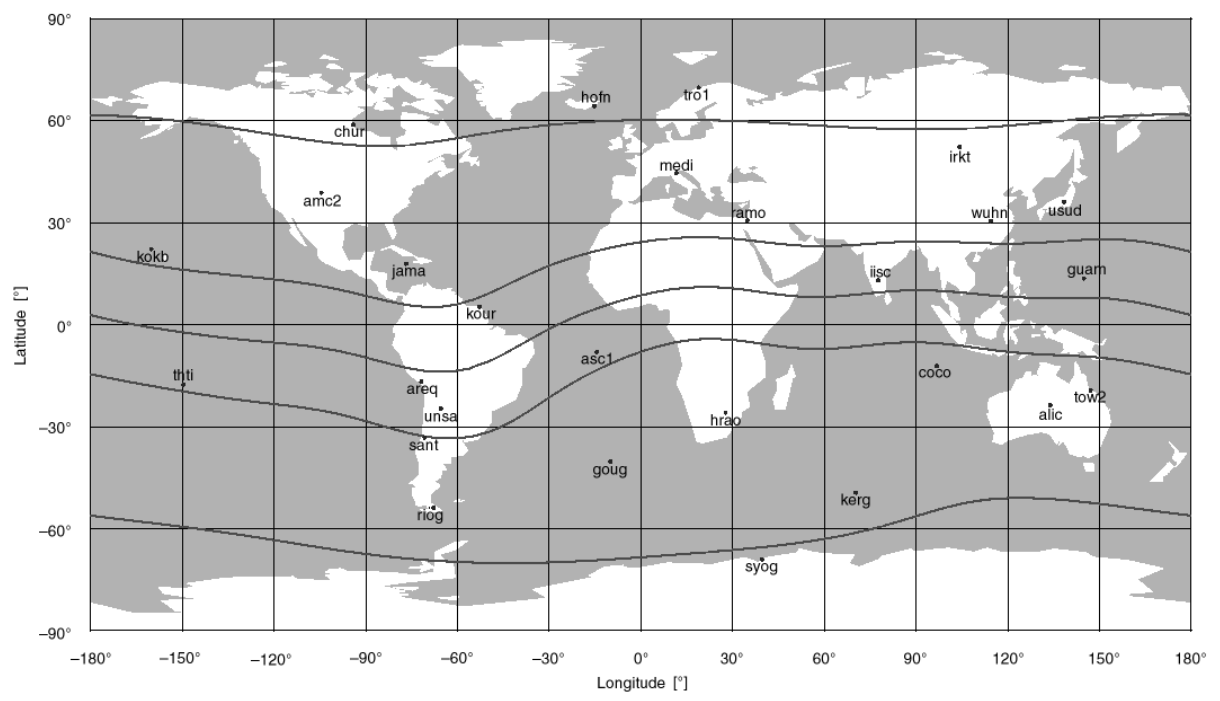

Fig. 3. Location of the IGS stations homogeneously distributed used to perform a concise analysis of the reconstruction technique performances for the day 27 March 2000 . Modip isolines at $-60^{\circ},-30^{\circ}, 0^{\circ}, 30^{\circ}, 60^{\circ}$ are indicated. 

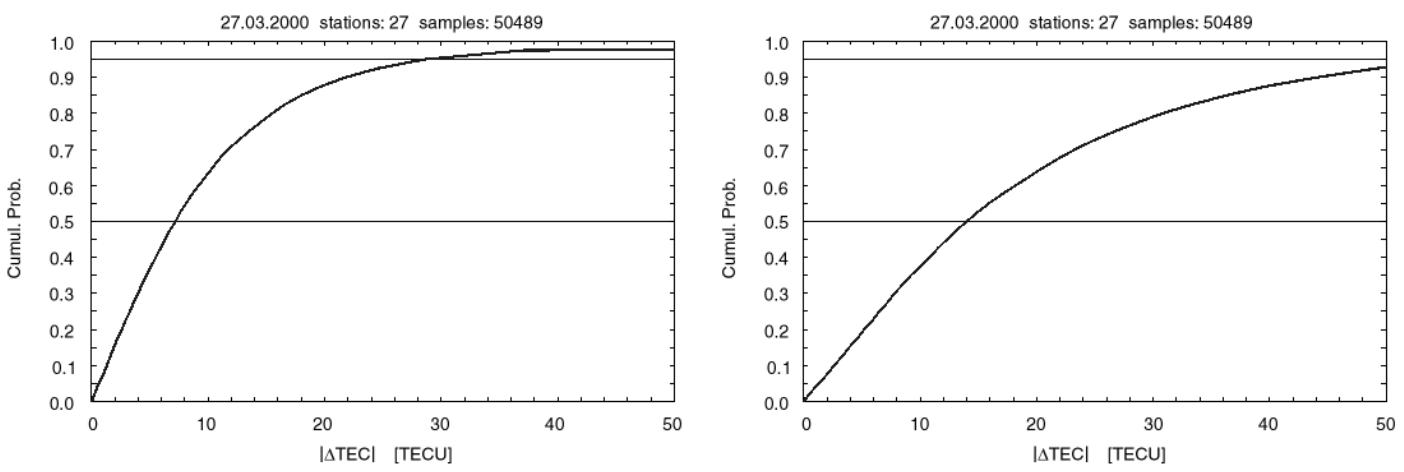

Fig. 4. Cumulative distributions of the absolute values of the differences between experimental and reconstructed slant TEC data corresponding to 27 IGS stations homogeneously distributed in Modip, for the day 27 March 2000. In the first case (left panel) the modeled slant TEC were reconstructed using NeQuick driven by $A z$ grids derived from La Plata vertical TEC maps. In the second case (right panel) the modeled slant TEC data were calculated using NeQuick driven by the solar flux of the day.

the Earth, whereas in the same hemisphere a latitudinal dependence of the errors magnitude can be observed: the agreement between experimental and reconstructed slant TEC is worse for stations with low Modip than for the other regions. This would indicate a lower effectiveness of the reconstruction method at low latitudes, as could be expected because of the complexity and high variability of the ionosphere at low latitudes. Nevertheless it must be noted that a part of this performance reduction could be related to a poor data coverage and lower experimental data quality for low-latitude regions.

As a final step, in order to have a concise indication of the reconstruction technique performances at global level, 27 IGS stations homogeneously distributed in Modip (see fig. 3) were selected and the corresponding experimental and reconstructed slant TEC data (using $\mathrm{NeQuick}$ driven by $A z$ grids derived from $\mathrm{La}$ Plata vertical TEC maps) were used to compute the cumulative distribution of the absolute value of the errors for the reference day. The results are shown in the left panel of fig. 4, where the cumulative distribution of the absolute value of the errors is plotted together with the lines corresponding to 50 and 95 percentiles. In this case the 95 percentile of 28.6 TECU represents the global performances of the reconstruction technique for the reference day.
The same experimental data set was used with modeled slant TEC obtained using NeQuick driven by the $10.7 \mathrm{~cm}$ solar radio flux corresponding to the day 27 March 2000 as given by the Space Environment Center (http://www.sec.noaa.gov). The corresponding cumulative distribution of the absolute value of the errors was computed and is shown in the right panel of fig. 4. In this case, the 95 percentile is 56.1 TECU and represents the global performances of NeQuick model for the reference day, without any adaptations derived from TEC data ingestion. Therefore the comparison of the cumulative distributions in fig. 4 gives an indication of the potentiality of the reconstruction technique based on model adaptation through actual data. For the reference day, the slant TEC errors at 95 percent level are reduced to a half of those obtained with the standard use of the Nequick model.

\subsection{Vertical incidence parameters}

Experimental peak parameters obtained from worldwide distributed ionosondes were used to test the capability of reconstruction method to reproduce peak parameters values on the basis of $A z$ grid calculation. An exhaustive analysis is given in (Miró Amarante et al., 
2003) and, as a further example, the comparison between hourly $f_{o} F_{2}$ measured at the locations indicated in table II on 27 March 2000 and the same values reconstructed with NeQuick model driven by $A z$ grids derived from La Plata maps is presented in fig. 5. As can be seen from the linear fit shown in the plot, there is a good agreement between experimental and recon-

Table II. Ionosondes used to validate the electron density reconstruction technique based on NeQuick model driven by $A z$ grids derived from La Plata vertical TEC maps for the day 27 March 2000.

\begin{tabular}{ccc}
\hline \hline Ionosonde & $\begin{array}{c}\text { Ionosonde } \\
\text { Lat N }\left(^{\circ}\right)\end{array}$ & $\begin{array}{c}\text { Ionosonde } \\
\text { Long E }\left(^{\circ}\right)\end{array}$ \\
\hline Bermuda & 32.4 & -64.7 \\
Dyess & 32.5 & -99.7 \\
Millstone Hill & 42.6 & -71.5 \\
Puerto Rico (Ramey) & 18.5 & -67.1 \\
Wallops Island & 37.9 & -75.5 \\
Fairford & 51.7 & -1.5 \\
Goose Bay & 53.3 & -60.3 \\
Narssarssuaq & 61.2 & -45.4 \\
Sondrestrom & 67.0 & -51.0 \\
Qaanaaq & 77.5 & -69.4 \\
\hline
\end{tabular}

structed $f_{o} F_{2}$. Therefore, even if the same degree of accuracy cannot be expected at all geographical locations, an attempt to reconstruct global $f_{o} F_{2}$ maps using only GPS derived TEC

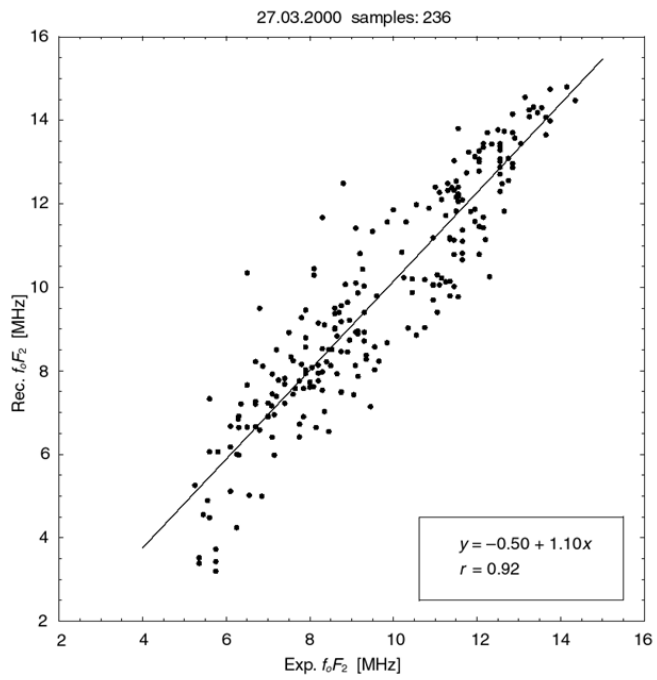

Fig. 5. Measured and reconstructed $f_{o} F_{2}$ using NeQuick model driven by $A z$ grids derived from La Plata vertical TEC maps for the day 27 March 2000. The best fit-line is also indicated.

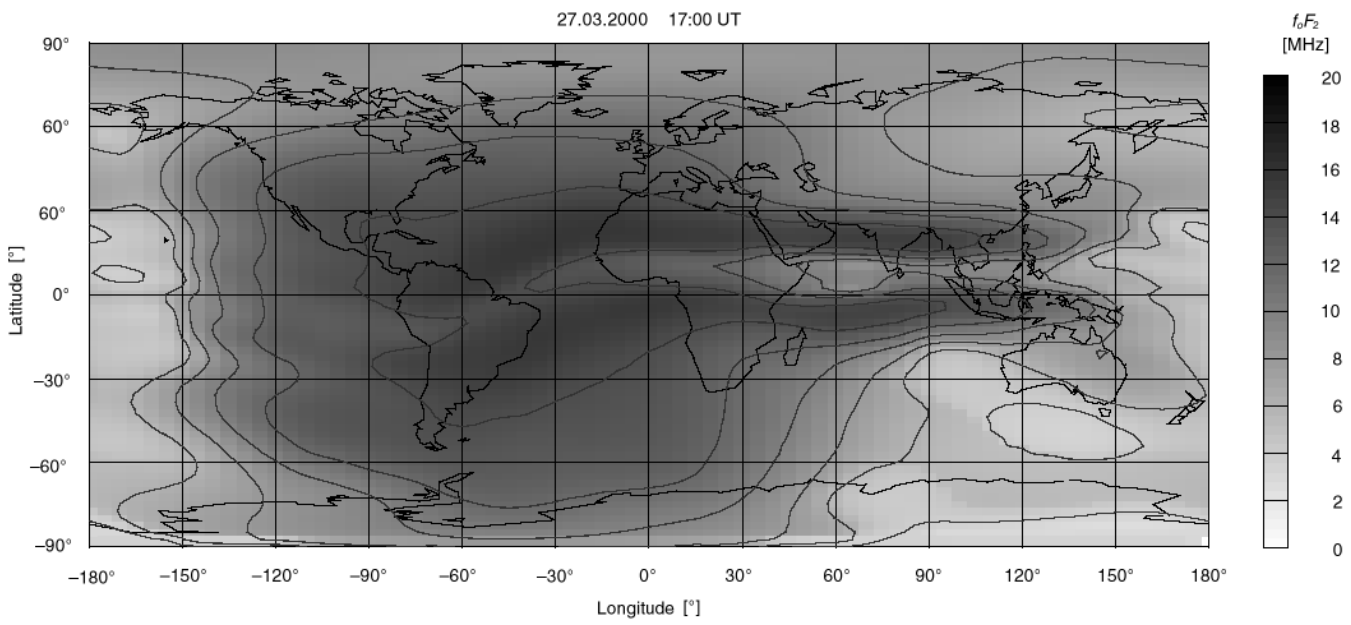

Fig. 6. Example of global $f_{o} F_{2}$ map reconstructed using NeQuick model driven by $A z$ grids derived from La Plata vertical TEC maps for the day 27 March 2000, 17:00 UT. 
data has been made. As an example, fig. 6 illustrates a reconstructed worldwide $f_{o} F_{2}$ map for the day 27 March 2000, 17:00 UT.

\section{Conclusions}

A model assisted reconstruction technique, able to provide a realistic 3D representation of the electron density of the ionosphere under given conditions, has been developed. It is based on experimental vertical TEC data ingestion into the NeQuick model: vertical TEC maps are used to retrieve worldwide grids for the model driving parameter $A z$. These $A z$ grids are computed at any epoch for which a vertical TEC map is available and, under particular conditions, the $A z$ grids can be interpolated in time to provide new grids for epochs when vertical TEC maps are not available. The computation of an $\mathrm{Az}$ grid allows to calculate the electron density of the ionosphere all over the world with the NeQuick model. As a consequence it is possible to calculate the TEC along any ground-to-satellite ray-path or to reconstruct global $f_{o} F_{2}$ maps. The reconstruction technique itself improves the model performances at global level when NeQuick is used to reconstruct experimental slant TEC values as it is demonstrated by the reduction to a half of the slant TEC errors at 95 percent level. The tests performed and the results obtained encourage further developments of the technique.

\section{Future developments}

At present the described reconstruction method is based on pre-calculated vertical TEC maps used to retrieve the $A z$ grid values that can drive NeQuick. It is important to observe that $A z$ grids can be calculated in several ways. A possible method to construct $A z$ grids, starting from experimental data, could be based on direct ingestion of slant TEC data. In this case a new approach should be followed in order to derive global $A z$ grid, starting from scattered slant TEC measurements. In addition, due to the flexibility of the NeQuick model, a reconstruction method based on a combined use of
$A z$ grids and experimental peak parameters values could be implemented.

\section{REFERENCES}

BILITZA, D. (2001): International Reference Ionosphere 2000, Radio Sci., 36 (2), 261-275.

BRUNINI, C. A. (1998): Global Ionospheric model from GPS measurements, Ph.D. Thesis (Facultad de Ciencias Astronómicas y Geofísicas, La Plata National University).

Di Giovanni, G. and S.M. Radicella (1990): An analytical model of the electron density profile in the ionosphere, Adv. Space Res., 10 (11), 27-30.

Hochegger, G., B. NAVA, S.M. Radicella and R. LEITINGER (2000): A family of ionospheric models for different uses, Phys. Chem. Earth, 25 (4), 307-310.

ITU (2001): Ionospheric propagation data and prediction methods required for the design of satellite services and systems ionosphere characteristics, Recommendation P.531-6, approved in 2001-02, managed by ITU-R Study Group SG3 (version 6 superseded in 2004 by version 7).

Komjathy, A., R.B. Langley and D. Bilitza (1998): Ingesting GPS-derived TEC data into the International Reference Ionosphere for single frequency radar altimeter ionospheric delay corrections, Adv. Space Res., 22 (6), 793-801.

Miró Amarante, G., S.M. Radicella, B. Nava and P. CoÏssON (2003): Validation of a new method for ionospheric electron density reconstruction by means of vertical incidence data, in Proceedings of 'Atmospheric Remote Sensing using Satellite navigation Systems', Special Symposium of the URSI Joint Working Group, Matera (FG), Italy, 335-342.

Nava, B., P. CoÏsson, G. Miró Amarante and S.M. RadiCELLA (2003): A new model assisted method for ionosphere electron density reconstruction, in Proceedings of 'Atmospheric Remote Sensing using Satellite navigation Systems', Special Symposium of the URSI Joint Working Group, Matera (FG), Italy, 343-348.

RADICELLA, S.M. and R. LeITINGER (2001): The evolution of the DGR approach to model electron density profiles, Adv. Space Res., 27, 35-40.

RAwER, K. (1963): Propagation of decameter waves (HFband), in Meteorological and Astronomical Influences on Radio Wave Propagation, edited by B. LANDMARK (Pergamon Press, New York), 221-250.

Schaer, S., G. Beutler, M. Rothacher and T.A. Springer (1996): Daily Global Ionosphere Maps based on GPS carrier phase data routinely produced by the CODE analysis center, in Proceedings of the IGS AC Workshop, Silver Spring MD, U.S.A.

Wang, C., G. HajJ, X. Pi, I.G. Rosen and B. Wilson (2004): Development of the Global Assimilative Ionospheric Model, Radio Sci., 39, RS1S06, doi: 10.1029/ 2002RS002854.

(received October 15, 2004 accepted March 09, 2005) 